\title{
Variants of the $T C F 7 L 2$ gene are associated with beta cell dysfunction and confer an increased risk of type 2 diabetes mellitus in the ULSAM cohort of Swedish elderly men
}

\author{
A. Dahlgren • B. Zethelius • K. Jensevik • \\ A.-C. Syvänen • C. Berne
}

Received: 10 January 2007 / Accepted: 16 May 2007 / Published online: 6 July 2007

(C) Springer-Verlag 2007

\begin{abstract}
Aims/hypothesis In a population-based cohort of elderly men with well-defined phenotypes and biochemical markers related to type 2 diabetes mellitus, we analysed two single nucleotide polymorphisms (SNPs), rs7903146 and rs12255372, in the transcription factor 7-like 2 gene (TCF7L2), which are associated with an increased risk of type 2 diabetes mellitus.

Materials and methods The 1,142 subjects were from the population-based Uppsala Longitudinal Study of Adult Men cohort study (see http://www.pubcare.uu.se/ULSAM/, last accessed in May 2007). Insulin sensitivity was assessed using a euglycaemic-hyperinsulinaemic clamp; fasting
\end{abstract}

Electronic supplementary material The online version of this article (doi:10.1007/s00125-007-0746-5) contains supplementary material, which is available to authorised users.

A. Dahlgren · A.-C. Syvänen

Molecular Medicine, Department of Medical Sciences,

Uppsala University,

Uppsala, Sweden

\section{B. Zethelius}

Department of Public Health and Caring Sciences/Geriatrics,

Uppsala University,

Uppsala, Sweden

K. Jensevik

Uppsala Clinical Research Center, Uppsala University,

Uppsala, Sweden

C. Berne

Endocrinology, Diabetes and Metabolism,

Department of Medical Sciences, Uppsala University,

Uppsala, Sweden

C. Berne $(\square)$

Department of Medical Sciences, University Hospital,

S-751 85 Uppsala, Sweden

e-mail: christian.berne@akademiska.se intact and 32-33 split proinsulin, immunoreactive insulin and specific insulin were measured in plasma samples. The SNPs rs7903146 and rs12255372 were genotyped using a fluorescent homogeneous single base extension assay. The SNP genotypes were analysed against diabetes prevalence at age 70 using logistic regression and against quantitative biochemical measures using linear regression analysis.

Results We replicated the association with type 2 diabetes mellitus for both SNPs in this cohort of elderly males. The highest significant odds ratio $(2.15,95 \%$ CI $1.20-3.85)$ was found for SNP rs7903146. The odds ratio for SNP rs12255372 was 1.69 (95\% CI 1.20-2.39). Both TCF7L2 SNPs were found to be significantly associated with plasma proinsulin when adjusting for insulin sensitivity, both in the whole cohort and when the diabetic subjects were excluded. Analysis for fasting plasma insulin or insulin sensitivity did not give significant results.

Conclusions/interpretation The association between the risk alleles of the two SNPs studied and levels of proinsulin in plasma, identified when adjusting for insulin sensitivity using euglycaemic-hyperinsulinaemic clamp measurements in this study, is an important novel finding.

Keywords Impaired fasting glucose - Impaired glucose tolerance - Proinsulin - Single base primer extension - Single nucleotide polymorphisms $\cdot T C F 7 L 2$ - Transcription factor 7 like 2 gene · Type 2 diabetes mellitus · Uppsala Longitudinal Study of Adult Men

$\begin{array}{ll}\text { Abbreviations } \\ \text { IRI } & \text { immunoreactive insulin } \\ \text { LD } & \text { linkage disequilibrium } \\ \text { M/I } & \text { insulin sensitivity index } \\ \text { SNP } & \text { single nucleotide polymorphism } \\ \text { ULSAM } & \text { Uppsala Longitudinal Study of Adult Men }\end{array}$




\section{Introduction}

The search for genetic variants underlying type 2 diabetes mellitus has had limited success in the past $[1,2]$, until recently when Grant and colleagues showed a significant association between a microsatellite marker (DG10S478) located in the transcription factor 7-like 2 gene (TCF7L2) and the risk of type 2 diabetes mellitus [3]. The original association was found in an Icelandic cohort of type 2 diabetes mellitus patients and control subjects and was subsequently replicated in both Danish and American cohorts. In addition to the microsatellite marker DG10S478, the study identified several single nucleotide polymorphisms (SNPs) in TCF7L2 that were in linkage disequilibrium (LD) with DG10S478 and also significantly associated with type 2 diabetes mellitus. Grant et al. suggested that two SNPs (rs12255372 and rs7903146) [3] be analysed for replication of their finding in other populations. Since the original publication, a number of studies have confirmed the association between TCF7L2 and type 2 diabetes mellitus in European, Asian and American cohorts [4-15].

The objective of our study was to analyse the SNPs rs12255372 and rs7903146 in the Uppsala Longitudinal Study of Adult Men (ULSAM) cohort. The participants in ULSAM have been particularly well characterised for phenotypes related to type 2 diabetes mellitus and several key biochemical markers for type 2 diabetes mellitus have been measured [16]. Insulin sensitivity was determined at age 70 using the euglycaemic-hyperinsulinaemic clamp [17]. The extensive phenotypic data available in ULSAM allowed us to investigate the possible biochemical aspects of associations between $T C F 7 L 2$ variants and the increased risk of type 2 diabetes mellitus in more depth than previous studies. While our study was in progress, the risk alleles of the two SNPs were shown to be associated with decreased levels of insulin in plasma and to affect progression to diabetes in subjects with impaired glucose tolerance [10]. Based on these findings, it has been suggested that the increased risk of type 2 diabetes mellitus associated with TCF7L2 could be caused by impaired beta cell function affecting glucose-stimulated insulin secretion $[7,10,11,18]$.

\section{Materials and methods}

Participants ULSAM is a population-based cohort study of diabetes and cardiovascular disease in men (see http://www. pubcare.uu.se/ULSAM/, last accessed in May 2007). The study was initiated in 1970 when all men born between 1920 and 1924 and residing in Uppsala county, Sweden, were invited to a health survey, in which 2,322 men participated [19]. After 20 years, at around 70 years of age, the men were invited to a reinvestigation, which was performed from August 1991 to May 1995, with 1,221 of the original 2,322 subjects participating [20]. All DNA samples $(n=1,142)$ analysed in this study were collected from the 70 -year-old $(71 \pm 0.6$ years) cohort. The study was approved by the Ethics Committee of the Faculty of Medicine at Uppsala University and informed consent was obtained from all participants in the study.

Biochemical measurements The diagnosis of type 2 diabetes mellitus was made at age 70 according to the 1999 WHO criteria [21] with a $75 \mathrm{~g}$ OGTT [16]. Insulin sensitivity was calculated as the glucose disposal rate divided by the mean plasma insulin concentration (where the glucose disposal rate is the concentration of glucose infused per unit of time per unit of body weight) $[16,17]$. Plasma samples were also analysed for immunoreactive insulin (IRI) during the OGTT, fasting intact and 32-33 split proinsulin and specific insulin [16]. At baseline (age 50) an IVGTT had been carried out using a glucose dose of $0.5 \mathrm{~g} / \mathrm{kg}$ body weight in $77.2 \%$ of the subjects in the original cohort. The serum IRI concentrations during the IVGTT were measured in duplicate blood samples drawn before the glucose injection. The early insulin response was expressed as the mean value of the serum insulin concentrations at 4, 6 and 8 min after start of the glucose injection.

Genotyping Two SNPs were selected (rs12255372 and rs7903146) for genotyping based on the recommendations made by Grant et al. for replicating their findings that TCF7L2 conferred risk of type 2 diabetes mellitus [3]. The selected SNPs were genotyped using a homogeneous single base extension assay with fluorescent polarisation detection [22] using in-house reagents. Fluorescence polarisation was recorded using an analysing device (Analyst AD; Molecular Devices, Sunnyvale, CA, USA). The genotype call rate of SNPs in the samples was $98 \%$ for both SNPs, with an accuracy, as determined by duplicate genotyping in independent experiments, of $100 \%$ according to $186(8 \%)$ genotype comparisons for the SNP rs12255372 and 160 (7\%) genotype comparisons for the SNP rs7903146. The genotypes conformed to Hardy-Weinberg equilibrium according to a $\chi^{2}$ test $(p>0.05)$.

Statistical analysis The statistical analyses were performed using SAS version 9.1 (SAS Institute, Cary, NC, USA). Genotype and allele frequencies for the SNPs were tested against diabetes (prevalence in the ULSAM cohort) as a dichotomous trait using logistic regression. For the quantitative biochemical measures, linear regression analysis was performed after excluding subjects with type 2 diabetes mellitus. For analysis including diabetic subjects, see Electronic Supplementary Material (ESM) Tables 1 and 2. Age was used as a covariate in all analyses. Adjustments 
were made for the influence of insulin sensitivity using either euglycaemic-hyperinsulinaemic clamp measurements or BMI. All subjects receiving insulin by injections were excluded from the analysis of biochemical measurements that included diabetic subjects. The nominal significance level was 0.05 . Correction for multiple testing using the effective number of SNPs and taking into account the LD between them resulted in a required critical $p$ value of 0.033 for significance [23]. No correction was made for the multiple biochemical traits tested. Significant findings from the linear regression analysis were further analysed by two-tailed $t$ testing on the mean values of biochemical measurements based on genotype.

\section{Results}

Logistic regression analysis of both TCF7L2 SNPs showed a significant association with type 2 diabetes mellitus in the ULSAM cohort (Table 1). The highest significant odds ratio (2.15, 95\% CI 1.20-3.85) was seen for SNP rs 7903146 when comparing the TT against the CC genotype. Comparison of the $\mathrm{CT}$ against the $\mathrm{CC}$ genotype also resulted in a significant odds ratio of 1.88 (95\% CI 1.32-2.67). An increased risk of type 2 diabetes mellitus was also observed for SNP rs12255372 with an odds ratio of 1.69 (95\% CI 1.20-2.39) when comparing the GT against the GG genotype. The TT genotype, compared with the GG genotype, did not yield a significant odds ratio $(1.52,95 \%$ CI $0.81-2.84)$. The LD between the SNPs was moderate $\left(D^{\prime}=0.833, r^{2}=0.675\right)$ based on the genotype data from the ULSAM cohort.

Table 1 Association analysis of SNP rs12255372 and rs7903146 genotypes with type 2 diabetes at age 70

\begin{tabular}{lllll}
\hline SNP & $\begin{array}{l}\text { Non- } \\
\text { diabetic }\end{array}$ & Diabetic & $\begin{array}{l}p \\
\text { value }\end{array}$ & $\begin{array}{l}\text { Odds ratio } \\
(95 \% \text { CI })\end{array}$ \\
\hline rs7903146 & & & & \\
CC & $496(0.56)$ & $67(0.40)$ & & CT vs CC \\
CT & $327(0.37)$ & $83(0.49)$ & 0.0006 & $1.88(1.32-2.67)$ \\
TT & $62(0.07)$ & $18(0.11)$ & & TT vs CC \\
& & & & $2.15(1.20-3.85)$ \\
T allele & $451(0.25)$ & $119(0.35)$ & 0.0002 & \\
C allele & $1,319(0.75)$ & $217(0.65)$ & & \\
rs12255372 & & & & GT vs GG \\
GG & $498(0.56)$ & $73(0.44)$ & & $1.69(1.20-2.39)$ \\
GT & $327(0.37)$ & $81(0.48)$ & 0.011 & TT vs GG \\
TT & $63(0.07)$ & $14(0.08)$ & & $1.52(0.81-2.84)$ \\
T allele & $453(0.26)$ & $109(0.32)$ & 0.0085 & \\
G allele & $1,323(0.74)$ & $227(0.68)$ & & \\
& & & &
\end{tabular}

Logistic regression was performed to test for association with type 2 diabetes mellitus. Diabetic subjects were diagnosed with type 2 diabetes mellitus in accordance with WHO criteria [21]
Analysis of SNP genotypes against biochemical measures was performed by linear regression analysis. Analysis of the non-diabetic group revealed a significant association for SNP rs7903146 with plasma proinsulin levels $(p=0.0049)$ when adjusting for age and insulin sensitivity (M/I) (Table 2). When adjusting for BMI, the association became non-significant $(p=0.18)$, but then an additional significant association was observed for a low IRI measured at $30 \mathrm{~min}$ of the OGTT $(p=0.03)$. Similar analyses for SNP rs12255372 showed significant associations for plasma proinsulin concentrations using $\mathrm{M} / \mathrm{I}$ as covariate $(p=0.004)$, but not for BMI $(p=0.12)$. A significant association was also shown for rs 12255372 and a low IRI measured at $30 \mathrm{~min}$ of the OGTT when adjusting for BMI $(p=0.03)$. Similar analyses for both SNPs in the whole cohort, including diabetic subjects, are depicted in ESM Tables 1 and 2.

Table 3 shows the significant findings, by two-tailed $t$ test, for rs7903146 and the mean concentrations of proinsulin and the $30 \mathrm{~min}$ oral insulin response to oral glucose according to genotype. The $\mathrm{T}$ allele of SNP rs7903146 was associated with higher plasma proinsulin concentrations when adjusting for $\mathrm{M} / \mathrm{I}(p=0.001)$ and also associated with significantly lower IRI levels at OGTT 30 min when adjusting for BMI $(p=0.01)$. Similar analyses for SNP rs12255372, as well as the mean values for all biochemical measures according to genotype in both subject groups, are shown in ESM Table 2. The distribution of mean values for the biochemical measures according to genotype for both SNPs are not shown here, but can be found in ESM Tables 3 and 4 .

Utilising the longitudinal data in ULSAM, we calculated odds ratios for development of IFG between age 50 and 70 . Comparison of the TT and CC genotypes of SNP rs7903146 resulted in an odds ratio of 2.55 (95\% CI $1.23-5.28 ; p=0.0094)$. The same analysis for $\mathrm{rs} 12255372$ resulted in two significant odds ratios: (1) when comparing the TT and GG genotypes of SNP rs 12255372 (odds ratio $3.25,95 \%$ CI 1.62-6.50; $p=0.0009$ ); and (2) when comparing the TT and GT genotypes (odds ratio 2.34, 95\% 1.15-4.72; $p=0.0183$ ).

In addition, in a subset of the cohort, measurements of early insulin response during an IVGTT [17, 24] performed at age 50 showed a lower insulin peak (mean 54.7 [SD 30.9] $\mathrm{mU} / 1, n=794$ ) in carriers of the TT genotype of SNP rs7903146, as compared with CC carriers (mean 68.4 [SD $50.8] \mathrm{mU} / 1, n=794)(p=0.004)$. This measure failed to reach significance $(p=0.13)$ for carriers of the TT genotype of SNP rs12255372, in whom the mean value was $58.8 \mathrm{mU} / 1$ (SD 30.6; $n=794$ ), as compared with GG carriers (mean $65.6 \mathrm{mU} / 1$ [SD 44.2]; $n=794$ ).

A correlation matrix for the biochemical measurements analysed in this study can be viewed in ESM Table 5. 
Table 2 Statistical analysis against quantitative biochemical measurements for all non-diabetic subjects at the age 70 investigation

\begin{tabular}{|c|c|c|c|c|c|c|}
\hline & Proinsulin & Split proinsulin & Insulin & OGTT IRI 0 min & $\begin{array}{l}\text { OGTT IRI } \\
30 \mathrm{~min}\end{array}$ & $\mathrm{M} / \mathrm{I}$ \\
\hline \multicolumn{7}{|l|}{ Study group } \\
\hline All & $8.4 \pm 8.3(999)$ & $10.8 \pm 11.6(999)$ & $51.6 \pm 36.8(1,005)$ & $76.8 \pm 42.7(1,032)$ & $341 \pm 218(1,004)$ & $4.7 \pm 2.3(1,000)$ \\
\hline Non-diabetic & $6.9 \pm 5.0(855)$ & $9.1 \pm 9.0(855)$ & $48.2 \pm 32.7(855)$ & $72.4 \pm 38.2(878)$ & $353 \pm 224(855)$ & $5.1 \pm 2.2(849)$ \\
\hline Diabetic & $16.8 \pm 15.7(144)$ & $21.0 \pm 18.0(144)$ & $71.9 \pm 50.9(146)$ & $101.6 \pm 56.8(154)$ & $271 \pm 167$ (149) & $2.7 \pm 1.6(151)$ \\
\hline \multicolumn{7}{|l|}{$\begin{array}{l}\text { SNP } \\
\text { rs7903146 }\end{array}$} \\
\hline $\mathrm{M} / \mathrm{I}$ & $0.005(816)$ & $0.21(816)$ & $0.67(820)$ & $0.91(839)$ & $0.27(842)$ & - \\
\hline $\begin{array}{l}\text { BMI } \\
\text { rs12255372 }\end{array}$ & $0.18(851)$ & - & $0.06(855)$ & $0.49(873)$ & $0.03(850)$ & - \\
\hline $\mathrm{M} / \mathrm{I}$ & $0.004(818)$ & $0.44(818)$ & $0.89(822)$ & $0.93(841)$ & $0.09(844)$ & - \\
\hline BMI & $0.12(854)$ & - & $0.23(858)$ & $0.50(876)$ & $0.03(853)$ & - \\
\hline
\end{tabular}

Values for biochemical measurements are means $\pm \mathrm{SD}$, with number $(n)$ of observations in parentheses. Euglycaemic-hyperinsulinaemic clamp measurements $(\mathrm{M} / \mathrm{I})$ values provided as $100 \times(\mu \mathrm{mol})(\mathrm{kg} \text { body weight })^{-1} \mathrm{~min}^{-1}(\mathrm{pmol} / \mathrm{l})^{-1}$; all other biochemical measurements are given as $\mathrm{pmol} / \mathrm{l}$. All analyses used age as a covariate in addition to M/I or BMI. Subjects with type 2 diabetes mellitus were excluded $(n=150)$

\section{Discussion}

The association between the TCF7L2 gene and type 2 diabetes mellitus is fast becoming a landmark achievement in genetic studies on human complex traits and diseases. Our study, along with many others, has successfully replicated the findings of Grant and colleagues regarding the significant association between the TCF $2 L 2$ and type 2 diabetes mellitus [3]. We also found that the high-risk alleles for both SNPs, particularly TT homozygotes, were associated with a significantly increased odds ratio to convert from normal fasting glucose to IFG between age 50 and 70. To further investigate the possible mechanisms underlying the association between TCF7L2 and type 2 diabetes mellitus, we utilised the unique phenotypic characteristics available in the ULSAM cohort. The euglycaemic-hyperinsulinaemic clamp measurements

Table 3 Analysis of SNP genotypes for significant findings from linear regression analysis using biochemical measurements from nondiabetic subjects at the age 70 investigation

\begin{tabular}{llll}
\hline SNP & $\begin{array}{l}\text { Proinsulin } \\
(\text { pmol/l })(n)\end{array}$ & $\begin{array}{l}\text { Two-tailed } t \text { test } \\
\text { comparison }\end{array}$ & $\begin{array}{l}\text { Proinsulin } \\
p \text { value }\end{array}$ \\
\hline rs7903146 & & & \\
CC & $6.8 \pm 5.0(479)$ & CC vs CT & 0.19 \\
CT & $6.9 \pm 4.1(313)$ & CT vs TT & 0.02 \\
TT & $8.3 \pm 8.2(59)$ & CC vs TT & 0.001 \\
rs12255372 & & & \\
GG & $6.8 \pm 5.0(475)$ & GG vs GT & 0.52 \\
GT & $6.9 \pm 4.2(316)$ & GT vs TT & 0.004 \\
TT & $8.4 \pm 8.4(60)$ & GG vs TT & 0.0009 \\
\hline
\end{tabular}

Values for proinsulin are means $\pm \mathrm{SD}$. All analyses used age and euglycaemic-hyperinsulinaemic clamp measurements $(\mathrm{M} / \mathrm{I})$ as covariates. Subjects with type 2 diabetes mellitus were excluded $(n=150)$ provided means to very specifically adjust for insulin sensitivity and enabled us to focus on actual beta cell function, characterised by the $30 \mathrm{~min}$ insulin response to oral glucose and fasting proinsulin concentration.

A novel finding in our study was the clear association between the $\mathrm{T}$ alleles of both investigated SNPs and increased concentrations of proinsulin in plasma, when using M/I measured by the euglycaemic-hyperinsulinaemic clamp as covariate. This finding suggests that the increased risk of type 2 diabetes mellitus conferred by the TCF7L2 variants is associated with dysfunction in the production of insulin within the beta cells, since we were able to compensate for the otherwise significant association between insulin resistance and elevated fasting plasma proinsulin (ESM Table 5). Thus we could distinguish the hyperproinsulinaemia associated with impending beta cell failure from that associated with the increased functional load imposed by insulin resistance.

We observed a significant association between the $30 \mathrm{~min}$ insulin response during the OGTT after adjustment for BMI at age 70 years and SNP rs7903146, an association also shown in other studies [7, 10, 18]. Analysis of a subset of the cohort, using the early insulin response data from IVGTT at age 50 years, showed a significantly lower acute insulin response in carriers of the high-risk allele of SNP rs7903146. These two observations made at 20 years interval, both after intravenous and oral administration of glucose, emphasise the association between TCF7L2 genetic variants and first-phase insulin release. The results highlight the importance of impaired insulin secretion, which occurs decades before the clinical onset of type 2 diabetes mellitus in individuals at increased genetic risk [24]. WNT signalling through TCF7L2 is linked to the production of the incretin glucagon-like peptide-1 in intestinal endocrine L cells [25]. In line with previous 
reports [18], we found that insulin secretion stimulated by both oral and intravenous glucose is affected by the TCF7L2 risk alleles; a finding that implicates mechanisms other than failure of beta cell stimulus, e.g. associations with defects in beta cell differentiation and proliferation. No association was observed between TCF7L2 SNPs and BMI or $\mathrm{M} / \mathrm{I}$ determined by the euglycaemic-hyperinsulinaemic clamp, nor with fasting concentrations of specific insulin or IRI, corroborating the notion that this genetic variation has no effect on insulin sensitivity, which has also been observed in the majority of other studies using homeostasis model assessment of insulin resistance or other models for assessment of insulin sensitivity.

Our study showed a significant association and increased risk between the two SNPs rs12255372 and rs7903146 in the TCF $7 L 2$ gene and type 2 diabetes mellitus. Both SNPs gave significant $p$ values, with the highest increased risk of disease being found for the T allele of SNP rs7903146, a finding consistent with observations in other populations [4, 6, 9]. For SNP rs12255372 the odds ratio was significant when comparing the GT and GG genotypes, while SNP rs7903146 showed an increased risk of type 2 diabetes for carriers of the $\mathrm{T}$ allele, both in heterozygous and homozygous forms. This difference between the two SNPs could possibly be due to lack of power in our cohort caused by the relatively low number of men with type 2 diabetes mellitus.

In summary, we found associations between the $\mathrm{T}$ alleles of both SNPs in the TCF7L2 gene and type 2 diabetes mellitus, thus replicating the findings by Grant and colleagues [3] and later studies. Moreover, we present a novel finding of a significant association between both SNPs and the proinsulin concentrations in plasma, using insulin sensitivity assessed by the euglycaemic-hyperinsulinaemic clamp to allow a more precise analysis of the association with beta cell function measured as the early insulin response to oral or intravenous glucose. These findings highlight impairment of insulin secretion in the development of type 2 diabetes mellitus, but need to be replicated. Further clinical and molecular studies are required to fully understand the role of TCF $7 L 2$ in the pathology and development of in type 2 diabetes mellitus. The discovery of the association between TCF $7 L 2$ and type 2 diabetes mellitus is quickly establishing itself as one of the most important findings in the field of complex disease genetics and may not only lead to a greater understanding of the genetic components in type 2 diabetes mellitus, but also show the way for future investigations into the genetics of other complex traits and diseases.

Acknowledgements This study was supported by Uppsala University, the Swedish Diabetes Association (C. Berne, B. Zethelius), the Family Ernfors Fund (B. Zethelius), the Sehlander Fund (B. Zethelius), the
Thuring Fund (B. Zethelius), the Swedish Research Council (A.-C. Syvänen), the EC FP5 project GenomEUtwin (A.-C. Syvänen) and the Knut and Alice Wallenberg Foundation via Wallenberg Consortium North. Appreciation goes to our previous mentors and collaborators in ULSAM, the late H. Lithell and C. N. Hales. We appreciate the statistical advice given by head-statistician L. Berglund at the Uppsala Clinical Research Center.

Duality of interest The authors declare that there is no duality of interest associated with this manuscript.

\section{References}

1. Ridderstrale M, Parikh H, Groop L (2005) Calpain 10 and type 2 diabetes: are we getting closer to an explanation? Curr Opin Clin Nutr Metab Care 8:361-366

2. Zeggini E, McCarthy MI (2007) TCF7L2: the biggest story in diabetes genetics since HLA? Diabetologia 50:1-4

3. Grant SF, Thorleifsson G, Reynisdottir I et al (2006) Variant of transcription factor 7-like 2 (TCF7L2) gene confers risk of type 2 diabetes. Nat Genet 38:320-323

4. Zhang C, Qi L, Hunter DJ et al (2006) Variant of transcription factor 7-like 2 (TCF7L2) gene and the risk of type 2 diabetes in large cohorts of U.S. women and men. Diabetes 55:2645-2648

5. van Vliet-Ostaptchouk JV, Shiri-Sverdlov R, Zhernakova A et al (2007) Association of variants of transcription factor 7-like 2 (TCF7L2) with susceptibility to type 2 diabetes in the Dutch Breda cohort. Diabetologia 50:59-62

6. Scott LJ, Bonnycastle LL, Willer CJ et al (2006) Association of transcription factor 7-like 2 (TCF7L2) variants with type 2 diabetes in a Finnish sample. Diabetes 55:2649-2653

7. Saxena R, Gianniny L, Burtt NP et al (2006) Common single nucleotide polymorphisms in TCF $7 L 2$ are reproducibly associated with type 2 diabetes and reduce the insulin response to glucose in nondiabetic individuals. Diabetes 55:2890-2895

8. Humphries SE, Gable D, Cooper JA et al (2006) Common variants in the TCF $7 L 2$ gene and predisposition to type 2 diabetes in UK European Whites, Indian Asians and Afro-Caribbean men and women. J Mol Med 84:1-10

9. Groves CJ, Zeggini E, Minton J et al (2006) Association analysis of 6,736 U.K. subjects provides replication and confirms TCF7L2 as a type 2 diabetes susceptibility gene with a substantial effect on individual risk. Diabetes 55:2640-2644

10. Florez JC, Jablonski KA, Bayley N et al (2006) TCF7L2 polymorphisms and progression to diabetes in the Diabetes Prevention Program. N Engl J Med 355:241-250

11. Damcott CM, Pollin TI, Reinhart LJ et al (2006) Polymorphisms in the transcription factor 7-like 2 (TCF7L2) gene are associated with type 2 diabetes in the Amish: replication and evidence for a role in both insulin secretion and insulin resistance. Diabetes 55:2654-2659

12. Chandak GR, Janipalli CS, Bhaskar S et al (2007) Common variants in the TCF7L2 gene are strongly associated with type 2 diabetes mellitus in the Indian population. Diabetologia 50:63-67

13. Cauchi S, Meyre D, Dina C et al (2006) Transcription factor TCF7L2 genetic study in the French population: expression in human beta-cells and adipose tissue and strong association with type 2 diabetes. Diabetes 55:2903-2908

14. Horikoshi M, Hara K, Ito C, Nagai R, Froguel P, Kadowaki T (2007) A genetic variation of the transcription factor 7-like 2 gene 
is associated with risk of type 2 diabetes in the Japanese population. Diabetologia 50:747-751

15. Hayashi T, Iwamoto Y, Kaku K, Hirose H, Maeda S (2007) Replication study for the association of TCF7L2 with susceptibility to type 2 diabetes in a Japanese population. Diabetologia 50:980-984

16. Zethelius B, Hales CN, Lithell HO, Berne C (2004) Insulin resistance, impaired early insulin response, and insulin propeptides as predictors of the development of type 2 diabetes: a population-based, 7-year follow-up study in 70-year-old men. Diabetes Care 27:1433-1438

17. DeFronzo RA, Tobin JD, Andres R (1979) Glucose clamp technique: a method for quantifying insulin secretion and resistance. Am J Physiol 237:E214-E223

18. Munoz J, Lok KH, Gower BA et al (2006) Polymorphism in the transcription factor 7-like 2 (TCF7L2) gene is associated with reduced insulin secretion in nondiabetic women. Diabetes 55:3630-3634

19. Hedstrand H (1975) A study of middle-aged men with particular reference to risk factors for cardiovascular disease. Ups J Med Sci Suppl 19:1-61
20. Byberg L, McKeigue PM, Zethelius B, Lithell HO (2000) Birth weight and the insulin resistance syndrome: association of low birth weight with truncal obesity and raised plasminogen activator inhibitor-1 but not with abdominal obesity or plasma lipid disturbances. Diabetologia 43:54-60

21. Alberti KG, Zimmet PZ (1998) Definition, diagnosis and classification of diabetes mellitus and its complications. Part 1: diagnosis and classification of diabetes mellitus provisional report of a WHO consultation. Diabet Med 15:539-553

22. Chen X, Levine L, Kwok PY (1999) Fluorescence polarization in homogeneous nucleic acid analysis. Genome Res 9:492-498

23. Nyholt DR (2004) A simple correction for multiple testing for single-nucleotide polymorphisms in linkage disequilibrium with each other. Am J Hum Genet 74:765-769

24. Zethelius B, Byberg L, Hales CN, Lithell H, Berne C (2003) Proinsulin and acute insulin response independently predict type 2 diabetes mellitus in men-report from 27 years of follow-up study. Diabetologia 46:20-26

25. Yi F, Brubaker PL, Jin T (2005) TCF-4 mediates cell type-specific regulation of proglucagon gene expression by beta-catenin and glycogen synthase kinase-3beta. J Biol Chem 280:1457-1464 